\title{
A dynamical system approach to texel identification in regular textures
}

\author{
S.E. Grigorescu, Student Member, IEEE, and N. Petkov \\ Institute of Mathematics and Computing Science, University of Groningen. \\ P.O. Box 800, 9700 AV Groningen, The Netherlands \\ simona@iwinet.rug.nl, petkov@cs.rug.nl
}

\begin{abstract}
We propose a texture analysis method based on Rényi's entropies. The method aims at identifying texels in regular textures by searching for the smallest window through which the minimum number of different visual patterns is observed when moving the window over a given texture. The experimental results show that any of Rényi's entropies can be used for texel identification. However, the second order entropy, due to its robust estimation, is the most reliable. The main advantages of the proposed method are its robustness and its flexibility. We illustrate the usefulness and the effectiveness of the method in a texture synthesis application and we compare it with other structural approaches.
\end{abstract}

\section{Introduction}

Only rarely have real world objects surfaces of uniform intensity; most of the time they are textured. The word texture is used to refer to a number of commonly encountered visual patterns in natural scenes, such as foliage, grass, pebbles, clouds, etc. While there is no proper definition of texture, it is widely accepted that the term generally refers to a repetition of certain basic elements, sometimes called texels. The textures exhibiting such a repetition of basic primitives are commonly referred to as structural textures.

Structural texture analysis focused on identifying periodicity in texture or on identifying texels and their placement rules. Such methods are mainly based on Fourier analysis [10], cooccurrence matrices [11, 15], and autocorrelation functions $[7,5]$. While widely used, these methods prove to be fragile when it comes to natural texture analysis due to the imperfection in the regularity of these textures [7, 11].

Whether they deal directly with texel identification or they search for periodicity in texture, the structural texture analysis methods try to find the basic texture unit - the texel. This step is important for texture analysis not only in the context of structural approaches, it can also be used when other methods are employed for texture study. Next to being a tool for texture analysis $[5,11]$, texel identification is also useful in other fields such as texture synthesis [11], texture compression [11], and image database retrieval [7].

In this paper, we propose a texel identification method based on a concept borrowed from information theory Rényi's generalized entropies. Rényi's entropy family is one of the most popular generalizations of Shannon entropy [6]. Similar to Shannon entropy, it measures the amount of uncertainty in predicting the output of a probabilistic event [6]: when the uncertainty is reduced, the entropy decreases. This property is used in the method proposed here. More precisely, the texel identification method described in the following sections searches for the smallest window through which the minimum number of different visual patterns - different in a given sense - is observed when moving the window over a given texture. Such a window has the property of minimizing Rényi's generalized entropies.

Unlike most of the structural texture analysis methods, the method proposed here does not use cooccurrence matrices, Fourier analysis, or autocorrelation functions. These methods exhibit some drawbacks. For example, the cooccurrence matrices are expensive from computational point of view. The autocorrelation functions and Fourier analysis are sensible to slight distortions or noise in the analyzed textures. Our method, while being computationally tractable, is robust to distortions and noise. Another advantage of the proposed method is its flexibility. It can easily incorporate different texture models by simply adapting the way in which two visual patterns observed through two different windows are compared. For illustration, we compare here the gray level distribution in the considered windows.

\section{Rényi's generalized entropies}

Rényi’s generalized entropies were introduced in [14] as a family of measures that characterize the distribution of a random variable. They are defined as follows: if a random variable $\xi$ takes the values $\left[x_{i}\right]_{i=1 \ldots N}$ with probabilities $\left[p_{i}=P\left(\xi=x_{i}\right)\right]_{i=1 \ldots N}$, then the generalized entropy 
of order $q$ of $\xi$ is defined as

$$
H_{q}= \begin{cases}\frac{\log \sum_{i=1}^{N} p_{i}^{q}}{1-q} & \text { for } q \neq 1, \\ \lim _{q \rightarrow 1}\left(\frac{\log \sum_{i=1}^{N} p_{i}^{q}}{1-q}\right)=-\sum_{i=1}^{N} p_{i} \log p_{i} & \text { for } q=1, \\ -\log \max _{i=1 \ldots N}\left(p_{i}\right) & \text { for } q \rightarrow \infty .\end{cases}
$$

The zero order entropy counts the number of values $x_{i}$ for which $p_{i}$ is nonzero ${ }^{1}$. The entropy of order one is the Shannon entropy. The entropy of order two, also called the quadratic entropy [4], gives the probability that $\xi$ takes twice the same value. The order of the entropy can be seen as a weight of the contribution of each $x_{i}$ to the value of the entropy. Higher order entropies give higher weights to those $x_{i}$ that are more probable and minimize the contribution of the values with rare incidence - the outliers. For the limit case $q \rightarrow \infty$, the entropy $H_{\infty}$, also called the min-entropy [2], depends only on the probability of occurrence of the most frequent $x_{i}$.

\section{Rényi's generalized entropies of texture}

Let us consider an artificial texture image (Fig. 1) comprising a checkerboard pattern whose black and white blocks are squares $k \times k$ pixels wide. Such a texture can be described as consisting of square texels of size $2 k \times 2 k$. If we observe this texture locally, through a square shaped window of size $2 k \times 2 k$, we will see pieces of the texture that are, in fact, circularly shifted versions of the same pattern. A similar thing will happen if the size of the observation window is a multiple of $2 k \times 2 k$, i.e. $4 k \times 4 k, 6 k \times 6 k$, etc. However, if the size of the observation window is not a multiple of $2 k \times 2 k$, we see a number of distinct patterns, i.e. patterns that are not merely circularly shifted versions of each other. In general, the number of observed distinct patterns depends on the window size and on the complexity of the analyzed texture.

Let us call visual event a certain pattern that is seen through a square shaped observation window of a given size. When we observe the same pattern or patterns that are circularly shifted versions of each other, we say that we encounter identical visual events. Otherwise, we consider the patterns as different events. When moving a window over a texture image we encounter a number of events. The number of different visual events encountered and their probabilities of occurrence are characteristics of the analyzed texture and they can be used to identify the texture.

\footnotetext{
${ }^{1}$ When using the standard convention that $0^{0}=0$.
}

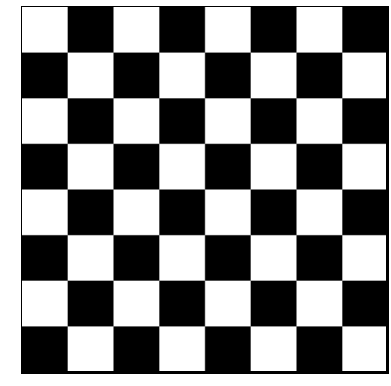

Figure 1. A synthetic texture.

A concise way of describing these characteristics is through Rényi's generalized entropies computed from the probability of occurrence of each distinct visual event - we call them Rényi's entropies of the texture.

Computing Rényi's entropies of a texture requires a definition of a visual event. Above, a visual event is defined as the set of all patterns that can be obtained from each other by circular shifting. More generally, we call a visual event the set of patterns that are similar in a given sense. For this purpose, we introduce a measure of pattern similarity. When such a measure classifies only identical patterns as the same visual event, the texture entropy is similar to Rényi's entropy of a random variable. When the classification measure labels patterns that are not identical, but rather similar, as the same visual event, Rényi's entropies of a texture come close to the entropies of a system as defined in dynamical system theory [6]. There, Rényi's entropies of a system are computed by covering an $n$-dimensional state subspace with $n$-dimensional disjoint volume elements of a given radius. Then, for each volume element, the probability that the state of the system falls in it is computed. Finally, based on these probabilities, the entropies are calculated according to (1). In texture entropy computation, using approximate pattern comparison methods is equivalent to covering the pattern space with volume elements not necessarily compact ones - and computing Rényi's entropies in the same manner as it is done for dynamical systems.

For a given texture, the texture entropies depend on the size of the observation window. In the followings, we study the behavior of the texture entropies as functions of the observation window size. For illustration purposes, we consider a simple case, the synthetic texture from Fig. 1. We compute the distribution of visual events observed in a square window of size $w \times w$ and we plot its corresponding $H_{q}(w)$ as a function of $w$.

From the family of generalized entropies we choose to study four representatives in order to see how the order affects the dependence of Rényi's entropy on the observation window size. We selected $H_{0}(w)$ because of its counting property. $H_{\infty}(w)$ is interesting because it shows how likely 


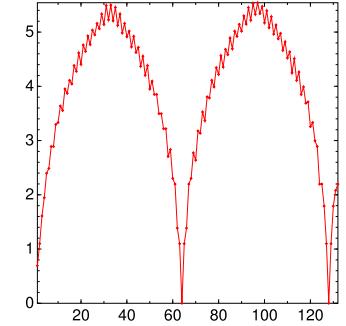

(a) $H_{0}(w)$

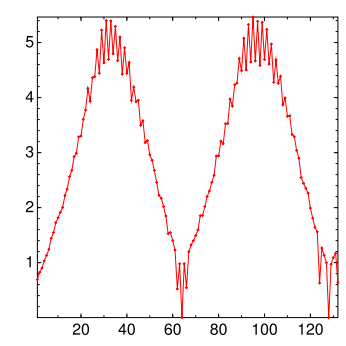

(c) $H_{2}(w)$

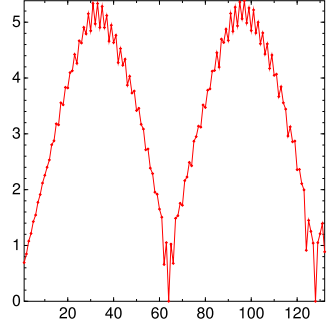

(b) $H_{1}(w)$

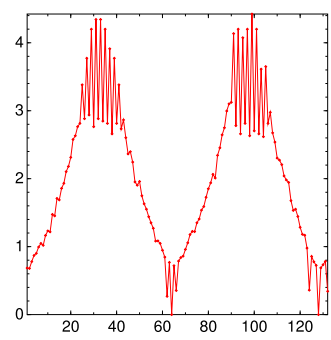

(d) $H_{\infty}(w)$
Figure 2. Rényi's entropies of the texture in Fig. 1, for $k=32$. The values on the abscissa represent the window size $w$, while the values on the ordinate represent $H_{q}(w)$.

it is to encounter the most frequent pattern. Further, we consider the Shannon entropy, $H_{1}(w)$, to see how informative it is compared with the other entropies and the quadratic entropy, $H_{2}(w)$, because it can be robustly estimated $[6,12]$.

We consider the pattern that can be observed through a window of size $w \times w$ as a probabilistic event. We compute $H_{q}$ as a function of $w, H_{q}(w)$, according to (1), where $p_{i}$ is obtained as the ratio between the number of occurrences of a given visual event in the analyzed image and the total number of visual events encountered in that image. For deciding whether two patterns represent the same visual event or not, we compare the histograms of the gray levels in the two observation windows. We consider two patterns as being the same event if the histograms associated with the two corresponding observation windows are identical.

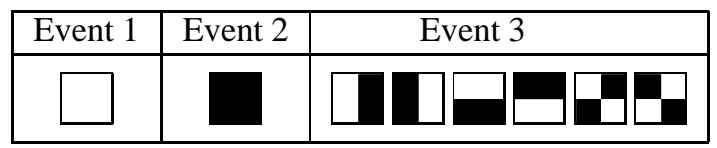

Figure 3. The three classes of patterns encountered in Fig. 1 when observed through a window of size $2 \times 2$.

The zero order entropy $H_{0}(w)$, Fig. 2a, indicates how many different visual events are encountered for a given window size. More precisely, $H_{0}(w)=\log M$ (see (1)), where $M$ is the number of distinct visual events observed in the analyzed texture. For example, for a window of size $1 \times 1(w=1)$, there are only two events that can be observed in the texture shown in Fig. 1 - a white pixel and a black pixel - leading to $H_{0}(1)=0.69$. For $w=2$, the eight patterns encountered in the analyzed texture are grouped in three visual events (see Fig. 3) leading to $H_{0}(2)=1.01$. When observed through a window of $2 \times 2$, six of the patterns encountered in Fig. 1 are considered the same visual event (see Fig. 3) because they share the same histogram.

While in a general context the classification of the patterns in the third column of Fig. 3 in the same group could be a mistake, in the context of texture analysis such a classification is not necessarily wrong since all these patterns could be regarded as translated or rotated versions of the same pattern. When classifying textures, assigning rotated or translated version of the same texture to one class is desirable. However, for segmentation purposes the lack of discrimination between texture patches that are rotated versions of each other is undesirable. Nonetheless, in the current context of texture entropy computation, the rotation and translation invariance can be added or removed by choosing an appropriate type of pattern comparison method for grouping different patterns in one event.

The zero order entropy reaches its minimum value when the observation window has a size multiple of $2 k \times 2 k$. Similarly, the other entropies plotted in Fig. 2 reach their minima for windows whose sizes are multiples of $2 k \times 2 k$. In other words, the window sizes for which the texture entropies reach their minima depend on the size of the texels of the texture and do not depend on the entropy's order.

\section{Rényi's entropies of natural textures}

The results displayed in Fig. 2 show that any of the Rényi's entropies of a texture could be used to find the texel size in a regular texture. The next question is how such entropies behave for natural textures. Unlike artificial textures, natural textures do not consist of identical texels, even when they are highly regular. In order to see how this difference affects Rényi's entropy behavior, we present experiments with textures from Brodatz album [1] and from VisTex texture database [16]. We selected a number of regular textures containing square texels of various sizes (see Fig. 4). The test images are of size $256 \times 256$ pixels.

To account for the variability of the texels in real textures, we compare the histograms of the patterns observed in two windows of the same size by means of the twosample Kolmogorov-Smirnov statistical test [8]. Applying Kolmogorov-Smirnov test to histogram comparison is a natural choice since histograms are estimates of the probability 


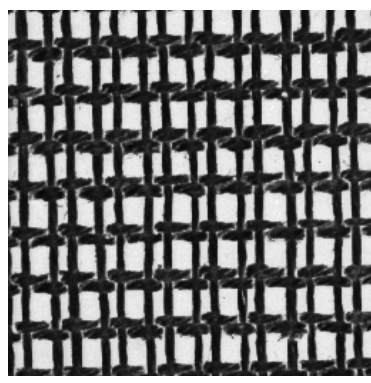

(a) $D 20$

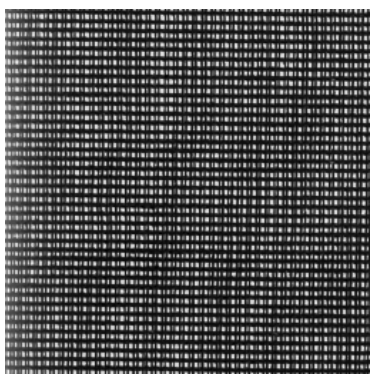

(c) $D 21$

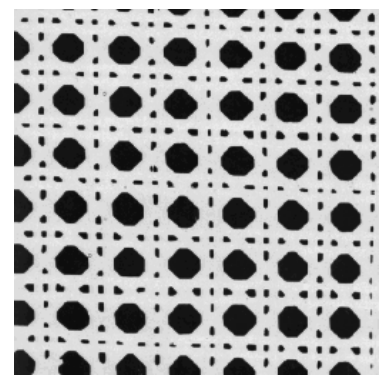

(b) $D 101$

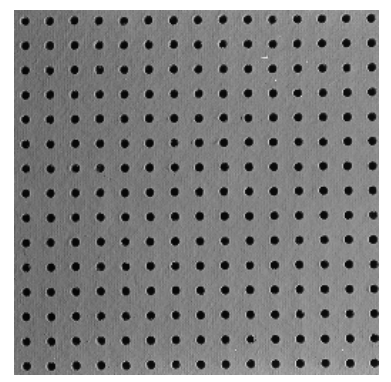

(d) Tile 9
Figure 4. Natural textures consisting of square shaped texels.

distribution functions of the gray levels.

In Fig. 5, we display the results obtained for the textures in Fig. 4. For conciseness reasons, we present here only the plots obtained for the quadratic entropy. Similar plots were obtained for the other entropies. These results were obtained using the Kolmogorov-Smirnov test at a significance level 0.01, a significance level often used in statistics.

In Section 3, we saw that in the case of a simple synthetic texture, Rényi's entropies can be used to determine the size of its texels, when the texels were square shaped. The entropies of such a texture are minimal around the window size that is equal to the size of the texel. Moreover, the entropy plots have deep valleys around such points.

Similar to the synthetic texture case, the generalized entropies of natural textures have deep valleys around the window size equal to the size of the texel. However, the texture entropies are not zero, neither are they minimal at such points. Typically, they do not reach the zero value at all and they are minimal for the smallest window size. Such a behavior has two causes: on one hand for small windows the number of all possible events is small leading to small entropy values even for irregular textures. On the other hand, for large windows the variability in the texels is so large that it leads to many false rejections in the classification of the encountered patterns and, consequently, to a large number of visual events, which means high entropy. Nonetheless, the sizes of the texels can be determined if one looks at the

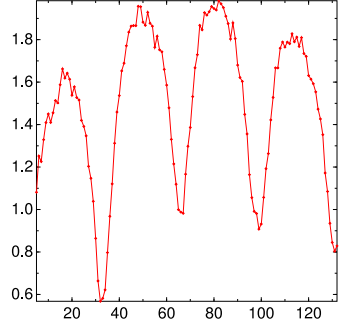

(a) D20: $w_{o}=33$

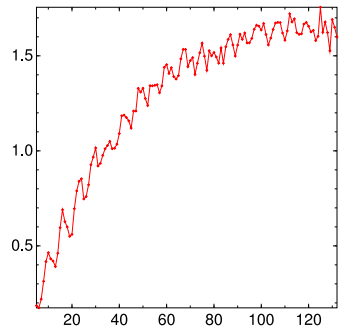

(c) D21: $w_{o}=6$

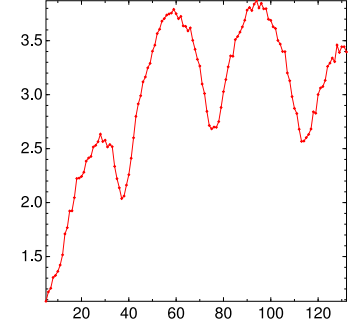

(b) D101: $w_{o}=37$

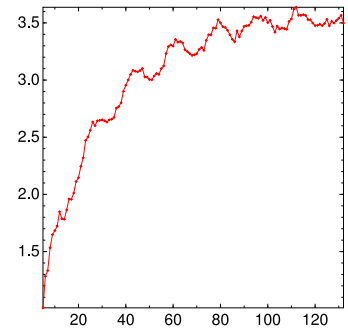

(d) Tile9: $w_{o}=14$
Figure 5. Rényi's quadratic entropies of the textures in Fig. 4. The caption of each plot gives the texture name and the size of the texel in the original texture as computed from that plot.

position of the deep valleys in the entropy plots. More precisely, the texel size is given by the window size for which the smallest local minimum ${ }^{2}$ is reached. The captions of each of the plots in Fig. 5 give the size of the texel as computed according to this rule.

\section{Application to texture synthesis}

In this section, we discuss an application of the texels size determination in texture synthesis by tiling. Such a synthesis method has the advantages of being fast and able to preserve the regularity of the texture sample. In Fig. 6, we present artificially generated counterparts of the textures in Fig. 4. Each artificial texture was generated by tiling with a square window randomly selected from the sample image. The size of that window was computed according to the method presented in Section 4.

\section{Summary, conclusions, and discussions}

In this paper, we study an application of Rényi's generalized entropies to texture analysis. More precisely, we show

\footnotetext{
${ }^{2}$ In the current context, $H_{q}(w)$ is considered to have a local minimum for window size $w$ if $H_{q}(w) \leq H_{q}(w-1)$ and $H_{q}(w) \leq H_{q}(w+1)$.
} 
that these entropies can be used to identify square shaped texels in regular textures. The proposed method is based on the property of the generalized entropies of the distribution of a stochastic variable of being small when the uncertainty in the outcome of that stochastic variable is small. In the case of texture analysis, the stochastic variable is the pattern observed through a square window of a given size. We show that, for regular textures with square shaped texels, Rényi's entropies depend on the size of the observation window and that they are small when the size of the observation window matches the size of the texels in the analyzed texture.

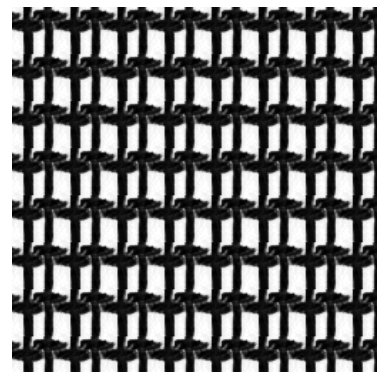

(a) D20: $w_{o}=33$

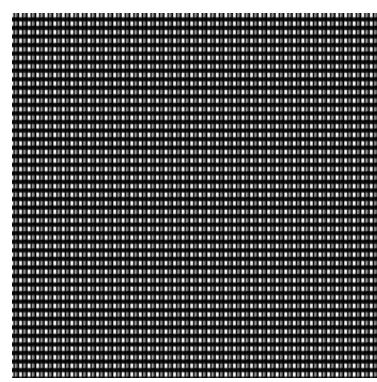

(c) D21: $w_{o}=6$

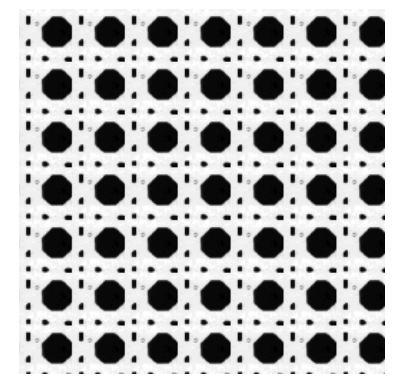

(b) D101: $w_{o}=37$

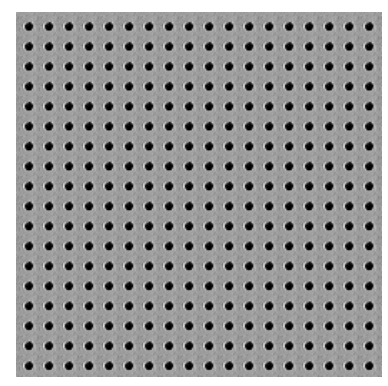

(d) Tile9: $w_{o}=14$
Figure 6. The synthesized counterparts of the texture in Fig. 4. The caption of each image gives the size of the texel used in the synthesis of that texture.

The experimental results obtained with our method show that any of Rényi's entropies can be used for texel identification. However, the second order entropy, due to its robust estimation, is the most reliable in combination with the straightforward valley seeking method employed here. Nonetheless, some of the other entropies remain interesting tools for texture analysis if more intricate estimation methods are used (see for example the method used in [4]).

The proposed method differs from previous work in structural texture analysis in that it does not use cooccurrence matrices, Fourier analysis, or autocorrelation functions. While methods based on the cooccurrence matrix give good results for textures such as the one in Fig. 4a (see for example the results reported in $[3,15])$, the same thing

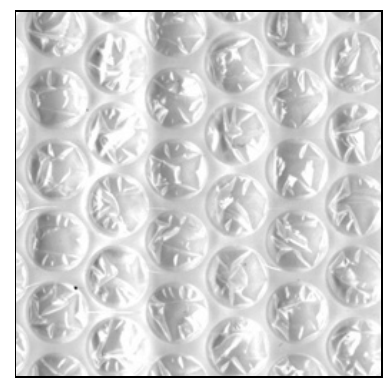

(a)

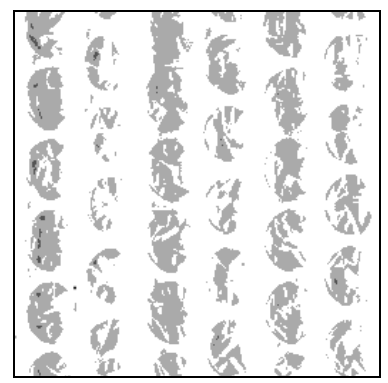

(b)
Figure 7. A natural texture (a) and its representation with four gray levels (b).

cannot be said about the results obtained for textures such as the one in Fig. 7a. The cooccurrence based methods, due to their computational requirements, use images with a reduced number of gray levels, typically 2 to 32 gray levels $[13,3,15]$. Such a reduction affects the periodicity of the analyzed texture (see Fig. 7b). When the number of gray levels used in the cooccurrence matrix computation is large enough to preserve the properties of the input images, the methods become computationally inefficient. The method proposed here overcomes the problems specific to the cooccurrence based methods. More precisely, it works on the full range of the gray values in the image while having a good computational efficiency.

The methods based on the autocorrelation function of an image search for prominent peaks of such a function (see for example the work reported in [5]). While this approach works easily for images such as the ones in Fig. $4 a$ and $4 b$, they will be less successful on textures such as the one in Fig. 7a. Fig. 8a shows the autocorrelation function of the texture in Fig. 7a computed according to (2). From this figure, it can be seen that the autocorrelation function does not have salient peaks in the vertical direction.

$$
[\rho(x, y)]_{x, y=0 \ldots 132}=\frac{\sum_{u=0}^{N} \sum_{v=0}^{N} I(u, v) I(u+x, v+y)}{\sum_{u=0}^{N} \sum_{v=0}^{N} I^{2}(u, v)}
$$

Similar to the autocorrelation based methods, the structural texture analysis approaches based on Fourier analysis [10] search for peaks, this time in the Fourier spectrum. Fig. $8 \mathrm{~b}$ represents the Fourier spectrum of the texture in Fig. 7a. Although a number of peaks can be seen in the Fourier spectrum, it is hard to identify those peaks corresponding to the horizontal and vertical periodicity existent in the analyzed texture.

The texture in Fig. 7a represents a difficult case to be 


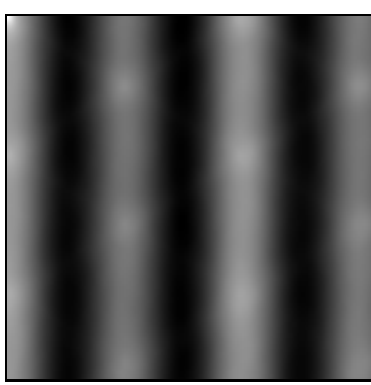

(a)

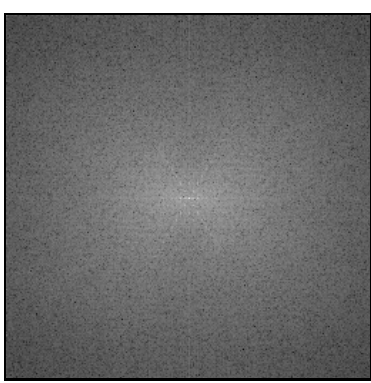

(b)

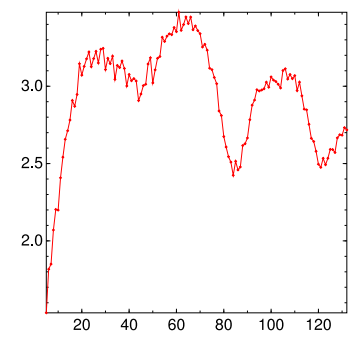

(c) $w_{o}=84$

Figure 8. The autocorrelation function (a), the Fourier spectrum (b), and Rényi's entropy (c) of the texture in Fig. $7 a$.

analyzed with structural methods because the high variability in the texture primitives. In general, structural methods such as the ones mentioned above assume that the analyzed texture is highly regular and that the presence of irregularities in the analyzed texture is incidental. In contrast, our approach, through the pattern comparison method, regards texel variability as an intrinsic feature of the texture.

The pattern comparison method used in our experiments is a statistical approach to texture analysis. It uses the gray level histogram of a window as a descriptor. The main reasons for such a choice are the invariance of the histogram to various transforms and its computational simplicity, while being a good discriminator between different textures. We are well aware of the limitations of such descriptors, however in the current context such limitations are not felt.

In the current setup, the proposed texel identification method is able to identify only square texels. This is due to the shape of the observation window used in the computation of pattern distributions. However, texels with different shapes can be identified if windows with different profiles are used. Our choice of a square window was inspired by the time delay embedding technique used in dynamical systems for time series analysis [6]. There, one 'embeds' the values from a given time series in a vector containing the last $n$ values preceding that value. Such a vector proved to be a better descriptor of the system that generated the time series [6] than a single scalar value. Moreover, computing Rényi's entropies starting from these vectors proved a successful approach to time series analysis characterization [9]. Using an extension of the time delay embedding for texture analysis is a suitable approach to texture analysis because texture is a neighborhood property. However, extending 1D techniques to 2D situations proves to be a difficult problem with no optimal solution. Here, we used the simplest way of extending a $1 \mathrm{D}$ technique to a $2 \mathrm{D}$ problem. Studying other extension alternatives remains a task for future work.

\section{References}

[1] P. Brodatz. Textures. Dover, 1966.

[2] C. Cachin. Smooth entropy and Rényi entropy. Lecture Notes in Computer Science, Advances in Cryptology: EUROCRYPT' '97, 1233:193-208, 1997.

[3] R. Conners and C. Harlow. Toward a structural textural analyzer based on statistical methods. Computer Graphics and Image Processing, 12(3):224-256, 1980.

[4] E. Gokcay and J. Principe. Information theoretic clustering. IEEE Trans. Pattern Analysis and Machine Intelligence, 24(2):158-171, 2002.

[5] S. Jan and Y. Hsueh. Window-size determination for granulometrical structural texture classification. Pattern Recognition Letters, 19(5-6):439-446, 1998.

[6] H. Kantz and T. Schreiber. Nonlinear Time Series Analysis. Cambridge Nonlinear Science series. Cambridge University Press, 1999.

[7] J. Leu. On indexing the periodicity of image textures. Image and Vision Computing, 19(13):987-1000, 2001.

[8] B. Lindgren. Statistical theory. Chapman \& Hall, fourth edition, 1993.

[9] I. Matsuba. Generalized information criterion for linear and nonlinear processes. Int. J. Bifurcation and Chaos, 12(2):389-395, 2002.

[10] T. Matsuyama, S. Miura, and M. Nagao. Structural analysis of natural textures by Fourier transformation. Computer Vision Graphics ans Image Processing, 25:347-362, 1983.

[11] G. Oh, S. Lee, and S. Y. Shin. Fast determination of textural periodicity using distance matching function. Pattern Recognition Letters, 20(2):191-197, 1999.

[12] T. S. Parker and L. O. Chua. Practical Numerical Algorithms for Chaotic Systems. Springer-Verlag, New York, 1989.

[13] J. Parkkinen, K. Selkäinaho, and E. Oja. Detecting texture periodicity from the cooccurrence matrix. Pattern Recognition Letters, 11(1):43-50, 1990.

[14] A. Rényi. On measures of entropy and information. In 4th Berkeley Symp.Math. Stat. and Prob., volume 1, pages 547561, 1961.

[15] V. Starovoitiv, S. Jeong, and R. Park. Texture periodicity detection: Features, properties and comparisons. IEEE Trans. Systems, Man and Cybernetics - Part A: Systems ans $\mathrm{Hu}$ mans, 28(6):839-849, 1998.

[16] Vision and M. group at the MIT Media Lab. Vision texture database. http://www-white.media.mit.edu/ vismod/ imagery/VisionTexture/ vistex.html. 